\title{
Crane location and selection by computer
}

COLIN GRAY

Department of Constructiuon Management

University of Reading, Whiteknights, Reading, UK

Cranes are used on nearly every building site as the primary means of materials movement, certainly during the critical early stages. Because of their cost it is prudent to optimize their use, such that on the well managed site the rate of work and the speed of the lifting operations becomes interdependant. The CRANES program has been developed to allow the rapid assessment of the lifting requirements implicit in a design by designers during the early design development. By the use of CAD and an expert system written in Prolog 2 this problem is approached. The output allows sensible evaluation of the problem by non experts at a time when the spacial and cost implications can be adjusted for.

Keywords: Cranes, Expert systems, CAD, Prolog 2, management, construction.

\section{Introduction}

The choice of crane for a building project is very dependant on the design of the building and therefore, the magnitude of the lifts and their location. Previous models (Furusaka and Gray, 1984) have typically approached the problem assuming that the building was of a regular geometric shape and suitable for conventional mathematical optimization. However, buildings are not always like that and a more flexible approach is needed to accommodate the vagaries of individual buildings. The more variable the building becomes the more important it is to be able to consider the effect on the work of the crane. The primary need is to ensure that the crane can cover the whole plan area, plus the pick up zone, with enough capability to lift all of the required loads safely. Secondly, the impact upon the progress of the work must be assessed because many of the critical operations will be crane dependant and, therefore, affected by the speed at which the crane can lift the loads. Selecting the right crane or combination of cranes is thus a quite complex problem. 


\section{The program}

The skills to evaluate the crane selection and its implication on the building operations are generally not available at a sufficiently early point in the design process to modify the design. Because of the interdependance of the crane and the work, optimization can only be achieved if the building design can be modified to ameliorate extremes in the crane specification. It is only by utilizing the principles of crane selection that the analysis can be undertaken at such an early stage. Equally the user is only seeking to determine: the type of crane and its broad cost; the effect on the work cycles and thus the project schedule, and the location and effect on access for load pick up and crane dismantling. The program, therefore, has been designed to consider the practical, technical, time and cost factors implicit in each type of crane and its application to the particular building.

\section{Computing environment and the use of computer graphics}

It was clear that the complexity of this program could best be handled by utilizing an approach based on AI computing languages. Our experience had indicated that Prolog was a suitable language for this type of program. The work commenced in 1985 and utilized the earliest available versions of Prolog which did not have a suitable graphics interface. In order to demonstrate the principles of the program a separate program written in GHOST was used to describe the relationship between the building's profile, the loads and the site area.

The user accessess the graphics program and is asked to describe the plan of the building and select a scale. In crane selection there are two types of load: a general lifting requirement and a specific load size and location. Once the outline is drawn the user is asked to locate the specific loads by moving the cursor to the correct position and typing in the value of the load at that point. The general loads are described by indicating the desired value at each extremety of the building. Loads in the pick up area can be indicated outside of the building footprint. The contouring facility within the package is then used to calculate lines of equal lift which are reproduced over the outline of the building. The user examines the output and locates a suitable position for the crane in an attempt to minimize the size of the crane, but at the same time considering the problems of access and dismantling. For each location, described as an area, the program will optimize the location and print out the radius of reach from that position to every load that has been indicated. This can be repeated for each location that has been chosen. The data is then transfered to the CRANES expert system, when required, in response to the questions that are asked.

This cumbersome system is currently being rewritten onto a PC to achieve an integrated approach. 


\section{The user interface}

Currently the dialog between the user and the program is through a series of question and answers. As described above the newer version will incorporate computer graphics, probably through a windowing system so that the user can see the effect of his decisions more easily. It is essential that graphics form a major component of this program because it is very difficult to describe all of the physical features of a building in words.

Output is in the form of the graphical layout of the building and a table of loads and radii for each location, additionaly there is a statement of each type of crane that satisfies the specification and its cost band. In this way the program provides the user with a guide as to the most suitable solution to the lifting problem. The analysis has been more thorough than conventionally available and has taken no more than twenty minutes to complete. Only the user can make the final choice.

\section{The inference engine}

To ease the complexity of analysis at this stage of the program's development only two types of crane are considered: tower cranes and mobile cranes. These are the predominant types used in the UK although it is recognized that there are other types used in other parts of the world, such as derricks in the USA.

This section of the program attempts to build a detailed specification for the crane from the craneage problem. It follows the sequence of analysis that would be used by an expert in this field. To demonstrate the process the case for a tower crane will be considered. The underlying assumption is that the cheapest crane is the one thats maximum lift at maximum radius is sufficient to provide the general lifting requirement over the full area required. Also, that the exceptional loads can be lifted within its normal load capacity profile. If the crane can: be erected by a crane standing next to it and dismantled in the same way; freestand on a rail base with no special foundations; work at the required rate and not impose on the programme of work, then it should be the cheapest. The inference engine through a series of questions seeks information about the tasks to be performed and the limitations which allow or inhibit the underlying assumption to be valid, so developing a specification for the particular problem.

The sequence of questions follows the following pattern, which is based on the detailed flow charts described more fully in Gray and Little (1985).

Impact upon the programme of work

It is important to determine whether one crane is sufficient to lift all of the required loads in the programme time available. Detailed information about 
the loads to be lifted is sought and used to calculate the basic floor cycle time for each of the buildings main floors. This is compared against generalised norms of performance for the type of structure and building. Where the crane cycle is longer than the norm the program, in the case of a concrete frame building, will supplement the crane with a concrete pump for the concrete handling and recalculate the cycle times. If the time still excedes the norm the program will offer the user the option to consider whether multiple cranes are required. Multiple cranes may well be required on grounds of reach or lifting capacity but this is usually secondary to the effect on the work rate.

\section{Free standing or tied in}

Cranes that are tied in affect the closure of the building and are generally disruptive to the programme of work. Ideally they should not be used. The problem may not be simply that of the building to be constructed, but that of the adjacent building over which the crane must traverse, either in the load carrying condition or in the free weather vane mode.

\section{Rail or fixed base}

The simplest base for a crane is to mount it onto a rail track with the counterballance weights on the base. Each piece can be recovered, which reduces the cost, and only a simple concrete foundation is required to spread the load. However, additional space is required for the track to be laid, even though the crane may not travel. Where this space is not available then the crane must have a specific foundation provided for it which also acts as the counterbalance. The normal procedure is to cast the bottom section of the crane into the foundation to ease the assembly process which means that it is written off. The restrictions of the site are therefore, an important determinant of the type of base such that, for a variety of factors, it may not be possible to locate the crane at the perimeter. Questions are asked about the areas available and the answers used to evaluate whether the rail based solution is possible or if not what the ground conditions are like so that the feasibility of a concrete foundation can be assessed.

\section{An internally located crane}

If the location of the crane external to the building is not viable then the crane could be located inside the plan area of the building. This has considerable implications and the user should return to the graphics and select the best position for the program to recalculate the loads and radii. In many cases lift shafts are used to minimize the penetration through the structure but where this is not possible then the structure will have to be left and built once the crane is removed. The biggest implication is the difficulty of removing the crane. This is a high cost operation and can be evaluated by using the program's knowledge of mobile cranes to calculate the correct size and thus the hire cost. 


\section{The data base}

At each point in the analysis the program searches the data base to find whether the crane specification can be matched by an available crane. If it can't then the analysis proceeds to the next stage in an attempt to reduce the size of the crane either by: tying it into the building; or locating it inside the building, or going to multiple cranes in the particular area.

The data base contains the specification of available cranes in the UK in terms of:

maximum reach

maximum load at maximum reach

maximum load

load carrying profile over the jib length

base size

rail base size

free standing height

height possible when tied in

free standing height above the highest tie

maximum unit size for dismantling

cost per week to hire

A cost evaluation is produced for each suitable type of crane.

\section{Conclusions}

The work described in this paper is a small section of a wider research programme examining the possibility of predicting construction time, which involves the consideration of cranage as the key plant item. In practice this is an extremely complex iterative process. However, there is a logic to the evaluation which, when coupled to the flexibility of Prolog, allows the generation of the expert system. The major gain is that the knowledge is transfered from the realm of a few, highly specialized people, into a form which is more generally available. The system is being developed to a second prototype to allow the concepts and principles to be more easily demonstrated on a PC. A fully developed form would require a larger data base of available cranes, parameters and costs.

\section{References}

Furusaka, S and Gray, C (1984) A model for the selection of the optimum crane for construction sites.

Construction Management and Economics, 2, 157-176

Gray, C and Little, J (1985) A systematic approach to the selection of an apppropriate crane for a construction site.

Construction Management and Economics, 3, 121-144 